kicker. The ovaries were cirrhotic and about half normal size. On the 26 th I received a note saying, "The mare has made a perfect recovery, and has been at work a week. She never showed the least outward symptom of having been operated upon." This improvement is maintained down to the present time.

No. I9. 8th February I902.-.-Polo pony, aged, excellent at her work, but squealed, kicked, and urinated profusely. Her tail swished continually from side to side, she was always in cestrum, and sometimes very unwilling and troublesome. The right ovary was about twice the normal size. Both contained a lot of Graafian vesicles. At the present time she seems perfectly cured of all her bad habits.

No. 20. 22nd March I902.-Brougham mare, eleven years old, in owner's possession six years. Worked satisfactorily until a year ago, when she commenced to kick, squeal, and urinate when put in harness. During the last three months she had been quite unmanageable, and at the present time it was only with difficulty that she could be approached in the region of the hind quarters.

The mare has made a good recovery from the operation, but it is too early to judge as to the effect on her temper and manners.

The ovaries were sent to Professor M'Fadyean, who pronounced them abnormally small and cirrhotic.

From the above twenty consecutive cases it will be seen that, under strict antiseptic precautions and chloroform anæthesia, the operation of ovariotomy is one which can be performed with comparatively little risk from peritonitis, the great bugbear of old-fashioned surgery. The immediate after effects, too, were practically nil, as in a large proportion of the cases the words used in describing the mare were, "The animal looks as if nothing had been done to her." It is quite a major operation, of abdominal surgery too, and yet the patients took not the slightest notice of it in any but the one instance (Case No. 2), in which the mare happened to be in foal. That Cases $I$ and $I 7$ were ridden within seven and ten days, respectively, clearly demonstrates the fact of the very slight ill effect.

Case 10 illustrates the fact that the removal of one ovary only will not cause oestrum to cease.

\title{
FURTHER EXPERIMENTS REGARDING THE IMMUNISATION OF CATTLE AGAINST TUBERCULOSIS*
}

\author{
By J. McFadrean, Royal Veterinary College, London.
}

IN an article which appeared in the preceding volume of this Journal (p. I 36), I déscribed certain experiments which had been carried out on four animals of the bovine species with the object of immunising them against tuberculosis. One of these animals had been killed and submitted to post-mortem examination, but the other three were still alive and apparently in good health at the time when the article was published. Since that date all three have died, and, let it be immediately stated, the cause of death in each case was tuber- 
culosis. The purpose of the present article is to record their later history, which presents some points of exceptional interest.

The details of the experiments carried out on these three cattle up to the month of June last are contained in the article referred to above, but for the better understanding of the subsequent experiments and their results readers will probably find it convenient if the later history of the animals is preceded by a summary of the facts already recorded.

\section{Case 1 .}

This animal, an Ayrshire heifer, was purchased at the end of September I 899, and it was then about a year and a half old. It was tested with tuberculin on the gth October following, and it reacted distinctly. When the test was repeated three days later it again reacted, but there was only an indecisive rise of temperature to a third test on the 27 th November. The animal was tested for the fourth time on the 8 th January 1900 , and there was again a distinct reaction.

Between the date of the last test and the month of June I9OI, the heifer received five intravenous inoculations of tubercle bacilli, as follows :-

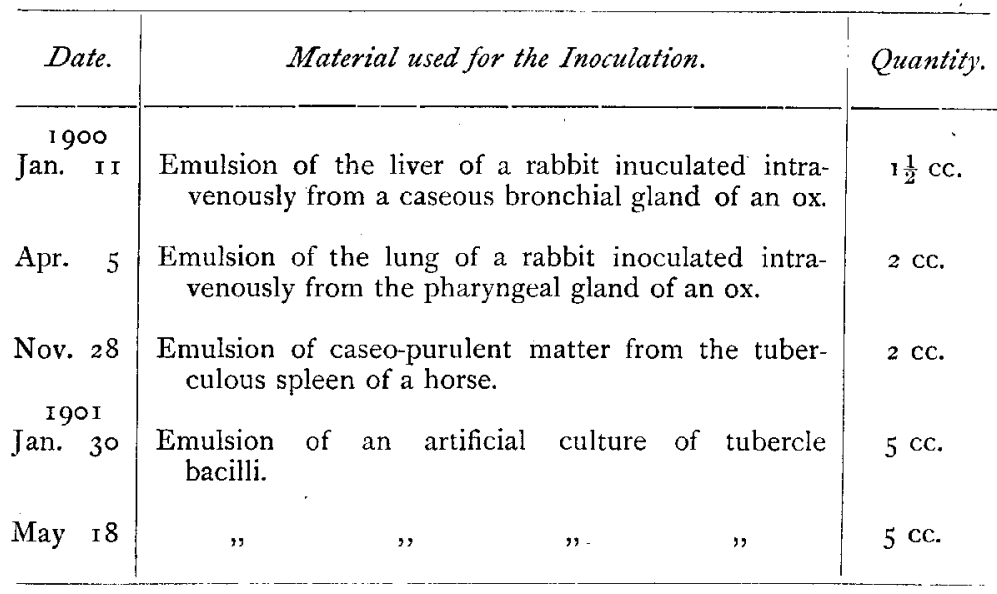

Having thus summarised the animal's earlier history, it remains to describe in detail the experiments that have been carried out since the month of June last.

Sixth Inoculation.-This was carried out on the 3 Ist July last. The material employed was an artificial culture of tubercle bacilli rubbed up with sterile water so as to form a turbid liquid. A coverglass preparation made with a trace of this liquid showed numerous tubercle bacilli, both singly and in clumps. The quantity of this liquid injected into the heifer's jugular vein was $5 \mathrm{cc}$.

At the same time two guinea-pigs were each inoculated subcutaneously with $\frac{1}{10} \mathrm{cc}$. of the same liquid. One of the guinea-pigs died on 26th August (twenty-six days), and the other on the 3rd September (thirty-four days), from a generalised tuberculosis. 
On the evening of the 3 Ist July, six hours after the intravenous inoculation, the heifer's temperature was $103: 3^{\circ}$, but on the following day it had fallen to $1008^{\circ}$, and the animal did not exhibit any abnormal symptoms during the following two months. It was tested once during that period ( 13 th September), and it did not react $\left(\max\right.$. temp. $\left.1032^{\circ}\right)$.

Seventh Inoculation. - On the 24th October last the heifer was again inoculated. On this occasion Io $\mathrm{cc}$. of a liquid rich in tubercle bacilli was injected into one of the jugular veins. The liquid was prepared by rubbing up a quantity of the rich surface growth from a bouillon culture of tubercle bacilli with sterile bouillon, and the number of bacilli present may be inferred from the fact that the liquid was opaque in a test tube I inch in diameter.

At the same time two guinea-pigs were inoculated, one intraperitoneally and the other under the skin of the abdomen, with $\frac{1}{10} \mathrm{cc}$. of the same liquid, and a control calf (eight months old) and a pony were inoculated intravenously, the former receiving $2 \mathrm{cc}$. and the latter $3 \mathrm{cc}$. of the liquid. Both of these animals had been tested with tuberculin on the 22 nd October and had not reacted.

It may be convenient here to interrupt the history of the heifer in order to complete that of the control animals.

Guinea-pigs.-The one inoculated intraperitoneally was killed on the I8th November last. A caseous nodule was present where the needle had penetrated the abdominal wall, the omentum was matted into a solid mass with tubercles, and the spleen was enlarged and filled with tubercles just visible to the naked eye.

The second guinea-pig (inoculated subcutaneously) died on the I $3^{\text {th }}$ January last, and the post-mortem examination showed the lesions of a generalised tuberculosis, involving the seat of inoculation, the precrural glands on both sides, the spleen, liver, and lungs.

Pony.-Immediately before the inoculation on the 24 th October the animals temperature was $997^{\circ}$, and three-and-a-half hours afterwards it had risen to IOI $8^{\circ}$. Subsequently the temperature became normal, and when the pony was tested with tuberculin on the 7 th November it reacted as follows:-

$\begin{array}{ccccc}\text { Time of Injection. } & \text { 6th Hour. } & \text { gth Hour. } & \text { I2th Hour. } & \text { 15th Hour. } \\ \mathrm{I00}^{\circ} 5 & \mathrm{IOI}^{\circ} 4 & \mathrm{IO}_{4} & \mathrm{IO}^{\circ} \mathrm{r} & \mathrm{IO}^{2} 2\end{array}$

Subsequently the temperature never exceeded $\mathrm{IO}^{\circ}$.

During the fortnight after inoculation the respiratory movements were irregular, varying from 36 to 16 per minute. After the 7 th November they were always hurried, and laterally about 40 per minute.

The pony was killed on the Igth November, and the post-mortem examination showed the lungs to be the seat of a miliary tuberculosis of extraordinary density. The tubercles were small, but quite visible to the naked eye, and their number was such that collectively they appeared to bulk more largely than the remnant of lung tissue between them (see annexed figure). Microscopic examination showed that many of the tubercles already contained giant cells, and that some of them had necrotic centres. Tubercle bacilli were present in considerable numbers, especially in the necrotic centres. The bronchial glands were about double the normal size, but tubercles were not visible 
in them with the naked eye, and no tubercle bacilli were detected in a scraping from the gland tissue. The abdominal organs were normal, save for the presence of a few doubtful points in the kidneys.

Calf.-Prior to inoculation the calf's temperature varied from $\mathrm{IO}_{3}^{\circ}$ to $104^{\circ}$, and during the following fortnight, it fluctuated between IOI $9^{\circ}$ and $104^{\circ} 1^{\circ}$. On the $14^{\text {th }}$ November it rose to $106^{\circ}$, and for a week afterwards it varied between $105^{\circ}$ and $104^{\circ} 2^{\circ}$. For a considerable

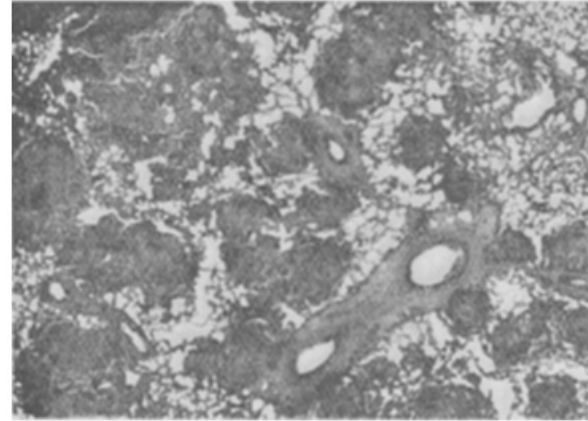

Section of lung of pony killed twenty-six days after intravenous inoculation with tubercle bacilli $(x 9)$.

time afterwards it continued to be unsteady, but for the last two months of its life it was generally under $102^{\circ}$, and (reactions excluded) it only once reached $103^{\circ}$. For a month after inoculation the respirations were generally abnormally frequent ( 24 to 42 per minute), but laterally they were quite normal.

Including the test before inoculation, the calf was tested six times with tuberculin, and the result on each occasion is shown below :-

Date. Time of Inoculation. 6th Hour. gth Hour. 12th Hour. 15th Hour.

$\begin{array}{rlllll}22 / 10 / 01 & 103.4 & 104 & 103.6 & 103 & 102.5 \\ 7 / 11 / 01 & 102.9 & 102.8 & 103 . & 104.6 & 104.8 \\ 29 / 11 / 01 & 102.3 & 107.2 & 104.6 & 106.2 & 105 \\ 6 / 1 / 02 & 103 & 103.4 & 105.4 & 103.6 & 103 \\ 3 / 3 / 02 & 101.6 & 102.2 & 102.8 & 105 & 104.6 \\ 22 / 3 / 02 & 100.8 & 103.4 & 103 & 101.6 & 102.2\end{array}$

The condition of the calf was rather poor at the time when it was inoculated, and during the next two months it sensibly deteriorated. On the other hand, it had somewhat improved during the last two months, though its growth appeared to be entirely checked.

It was killed on the 24 th March 1902, and the lesions discovered in it were as follows:-

At the seat of inoculation on the right side of the neck, between the skin and the jugular vein, there was a nodule about the volume of a walnut, but oval in shape, and composed of a fibrous sac enclosing thick caseo-purulent contents.

The whole of the lymphatic glands of the limbs, trunk, head, and neck were normal. The peritoneum and the whole of the abdominal viscera were normal. In one mesenteric lymphatic gland there was a partially calcified tubercle about the size of a common pin's head. The bronchial and mediastinal glands were normal in size, but three pin-head non-calcified tubercles were present in the latter. 
On the surface of the lungs there was a small amount of soft fleshy growth, at some places distinctly mushroom-shaped, and having the usual appearance of a commencing pleural tuberculosis. In the parenchyma of the lungs, by careful searching, a few (less than a dozen) firm, fibrous-looking, shotty tubercles, some larger than barley grains were detected. With this exception the lung tissue was perfectly normal. The brain and its membranes were normal.

Tubercle bacilli were easily detected in the caseo-pus of the lesion in the neck, and in the partially calcified tubercle from the mesenteric gland, but none were found in the thoracic lesions.

The history of the Ayrshire heifer subsequent to the inoculation on the 24 th October, may now be resumed.

Some hours after inoculation the animal's temperature had risen from $102 \cdot 2^{\circ}$ to $1062^{\circ}$, and during the next ten days it was unsteady, fluctuating between $102.8^{\circ}$ and $1043^{\circ}$. Afterwards it was with one or two exceptions quite normal (under $\mathrm{IO}^{\circ}$ ). Its respirations.were never seriously disturbed, and there was no loss of appetite or condition.

It was tested with tuberculin on the dates and with the results shown below (the first test was made two days before the last intravenous inoculation):-

Date. Time of Inoculation. 6th Hour. gth Hour. T2th Hour. I5th Hour.

$\begin{array}{rlllll}22 / 10 / 0 \text { I } & \text { IOI.6 } & \text { IO4.3 } & \text { IOI.7 } & \text { IOI.8 } & \text { IOI.6 } \\ 7 / 11 / 0 \text { I } & \text { IOI.6 } & \text { IO2.2 } & \text { IO0.8 } & \text { IOI } & \text { IOI.2 } \\ 29 / 11 / 01 & \text { IOI.5 } & \text { IO5.8 } & \text { 103.4 } & \text { IOI.8 } & \text { IOI.7 }\end{array}$

On the I6th December the animal, as usual, appeared to be quite well. It ate as usual, and its temperature was IOI $^{\circ} I^{\circ}$ (the highest temperature during the preceding fortnight had been $102^{\circ} 6^{\circ}$ ). During the early morning of the I 7 th December the heifer was found down in its loose-box, and it died almost immediately. Needless to say, its death was quite unexpected. The post-mortem examination was made on the forenoon of the same day, and the following is the record of it:-

Post-mortem Examination.-Body well nourished, abdomen slightly tympanitic. On the omentum there are a few small pedunculated perlsucht growths and also some diffuse growths of the same nature. Several of the mesenteric glands, although only very slightly enlarged, contain numerous tubercles about the size of a barley grain. These are firm and shotty, with fibrous capsules and caseous centres; none of them are sensibly calcified. Similar tubercles are also present in some of the lymphatic glands of the rumen. The interior of the stomach and intestines is free from tubercles, but there are some areas of congestion in the small intestine. The spleen and liver are apparently quite free from tubercles, but some firm, fibroid tubercles are present in the hepatic lymphatic glands, though these are not enlargẹd. Kidneys a little enlarged; capsule strips off readily. None of the lobules are normal, and some have their tissue almost entirely destroyed, their surface being of an almost uniform greyish colour. On section these are found to be tuberculous, and more or less caseous in both cortex and medulla. Other lobules have such patches but are not so extensively destroyed, and some of them show the dark-red cortex dotted with pin-head tubercles. The right and left kidneys are practically equally affected. At several places the lungs are united 
by fibrous adhesions to the chest wall and diaphragm. The bronchial and mediastinal glands are slightly enlarged, and all contain macroscopic tubercles in the cortex. For the most part the lung tissue is spongy, and apparently free from tubercles, but a considerable area towards the base of each lung, adjoining the thin diaphragmatic edge, when grasped is felt to be semi-solid. When cut into this is found to contain numerous tubercles, with a few collections of caseo-pus up to the size of a large pea; both lungs are similarly affected.

The following groups of glands all contain tubercles:-Both popliteal, both axillary, the right prescapular, and the right gland at the entrance to the pelvis; all these are about double the normal size, and their ground tissue is dark from congestion. Several of these glands have soft centres nearly the size of a vetch pea, with white caseopus. The glands of these groups not showing visible tubercles are also somewhat enlarged and congested. The pharyngeal glands are normal in size, and show no tubercles. The cardiac muscle and muscular masses of the body show no tuberculous lesions. The pia mater at the base of the brain, especially over the anterior part of the sides of the pons and medulla, is beset with pin-head tubercles.

Cover-glass preparations made from the pulmonary and renal lesions and from the tubercles in the pia mater showed considerable numbers of tubercle bacilli.

It appeared to be of interest to ascertain the degree of virulence possessed by the bacilli in these lesions, and, accordingly, on the Igth December, two guinea-pigs were inoculated subcutaneously with a trace of caseo-purulent material from one of the kidneys. One of these animals died with the lesions of a generalised tuberculosis on the I2th March, and the other when killed on the following day showed an identical condition.

\section{Case 11 .}

The subject of the experiment was a Hereford heifer, about a year old when the experiment was begun in May I 899 . On the 5 th of that month it was tested with tuberculin, and did not react. Between that date and the month of June last (which part of its history has already been recorded in the article previously referred to) tubercle bacilli were injected into its veins on eight occasions, as follows :-

[TABLE. 


\begin{tabular}{|c|c|c|}
\hline Date. & Material used for the Inoculation. & Quantity. \\
\hline $\begin{array}{c}\text { I } 899 \\
\text { May } 10\end{array}$ & Emulsion of the tuberculous liver of a pheasant. & $2 \frac{1}{2} \mathrm{cc}$. \\
\hline Nov. I I & $\begin{array}{l}\text { Emulsion of the liver of a rabbit inoculated intraven- } \\
\text { ously from the tuberculous mesenteric gland of a } \\
\text { horse. }\end{array}$ & $2 \frac{1}{2} \mathrm{cc}$ \\
\hline $\begin{array}{l}\text { I } 900 \\
\text { Jan. I I }\end{array}$ & Same as for Case I. on the same date. & $\mathbf{I} \frac{1}{2} \mathrm{cc}$. \\
\hline April 5 & , & $3 \mathrm{cc}$ \\
\hline $\begin{array}{l}\text { Nov. } 28 \\
1901\end{array}$ & " & $2 \mathrm{cc}$ \\
\hline Jan. 30 & " & $5 \mathrm{cc}$. \\
\hline Mar. 23 & Emulsion of an artificial culture of tubercle bacilli. & I $5 \mathrm{cc}$. \\
\hline May is & , & I5 cc. \\
\hline
\end{tabular}

Subsequent to the date of publication of the former article the heifer was inoculated intravenously on three occasions, the particulars of which must now be recorded.

Ninth Inoculation.-This was carried out on the 2 Ist July I90I, the material and the dose being the same as for the animal of last experiment on the same date, viz., $5 \mathrm{cc}$. of a rich emulsion of tubercle bacilli from an artificial culture. Six hours afterwards the heifer's temperature had risen to $105^{\circ} 7^{\circ}$, and it remained above normal for about a week afterwards. On the 13 th September the animal was tested with tuberculin, with the result that the temperature rose (from $1017^{\circ}$ ) to $104.6^{\circ}$ at the ninth hour, but fell to $\mathrm{IOI}^{\circ}$ at the fifteenth hour.

Tenth Inoculation.-On the 5 th October last the heifer received a tenth intravenous inoculation of tubercle bacilli. The material was prepared by rubbing up part of the surface growth of an artificial bouillon culture with sterile water, and the quantity of liquid injected was Io cc. The liquid was opaque in a test tube one inch in diameter, and a cover-glass preparation made from it showed great numbers of bacilli in every field. At the same time two guinea-pigs were inoculated subcutaneously with $\frac{1}{2} \overline{c c}$. of the liquid.

One of these guinea pigs was killed on the I 7 th November, and the post-mortem examination showed a caseating lesion in the abdominal wall at the seat of inoculation, enlargement and caseation of the adjacent precrural gland, and numerous tubercles in the spleen, liver, and lungs. The other guinea-pig died with the lesions of a generalised tuberculosis on the $24^{\text {th }}$ February last.

Three hours after inoculation the heifer's temperature had risen to $105^{\circ} 4^{\circ}$, and during the next four days it fluctuated between $10 I^{\circ} 7^{\circ}$ and $103^{\circ} 2^{\circ}$. After that it became normal (under $103^{\circ}$ ), and when the animal 
was tested with tuberculin on the 22nd October the result was as follows :-

$\begin{array}{ccccc}\text { Time of Injection. } & \text { 6th Hour. } & \text { gth Hour. } & \text { I2th Hour. } & \text { 15th Hour. } \\ 102.5 & 103.8 & 1023 & 102.5 & 102 \cdot 2\end{array}$

Eleventh Inoculation.-On the 24 th October the heifer was inoculated intravenously for the eleventh time. The details of the inoculation were exactly the same as for the subject of the preceding experiment on the same date ( $10 \mathrm{cc}$. of a rich emulsion of tubercle bacilli from an artificial culture).

Just before inoculation the temperature was $102 \%$, and four hours afterwards it had risen to $107^{\circ}$. On the following day it had fallen to $103 . I^{\circ}$.

For a week afterwards it varied from $10^{\circ} 23^{\circ}$ to $1037^{\circ}$, but except on the occasions of testing, after the $3^{\text {rd }}$ November it exceeded $103^{\circ}$ on only three days.

After the last intravenous inoculation the heifer was twice tested with tuberculin, as shown below :-

\begin{tabular}{|c|c|c|c|c|c|}
\hline$e$. & $\begin{array}{c}\text { Time of Injection. } \\
\text { 10 } 5 \text {. } 5\end{array}$ & $\begin{array}{l}\text { 6th Hour. } \\
\text { I04\% }\end{array}$ & $\begin{array}{c}\text { gth Hour. } \\
\text { I02.6 }\end{array}$ & $\begin{array}{l}\text { 12th Hour. } \\
\text { 102 }\end{array}$ & $\begin{array}{l}\text { 15th Hour } \\
\text { 102 }\end{array}$ \\
\hline & IOI & 104 & Io & rot 8 & - \\
\hline
\end{tabular}

Immediately after the inoculation on the $24^{\text {th }}$ October the respirations became notably increased in frequency, and the abnormality continued. On only one day (the 3 Ist October) were they below 30 per minute; once they were 70 , and on an average about 50 .

The heifer died suddenly and quite unexpectedly about 3 A.M. on the $23 \mathrm{rd}$ December last. On the previous day, save for the beforementioned abnormality of the respiratory movements, it had appeared to be quite well. It seemed quite lively, ate as usual, and had a temperature of $1021^{\circ}$.

The following are the notes of the post-mortem examination, which was made at noon on the 23 rd December :-

Post-mortem Examination.-Slight abdominal tympany. Rigor mortis well marked. Condition good; subcutaneous and intermuscular fat abundant. The udder and its lymphatic glands are normal. Subcutaneously, over the left jugular vein there is a dense fibrous nodule, about the size of two barley grains. Peritoneum normal. Fat in mesentery, omentum, and around kidneys abundant. Mesenteric glands normal. Liver and hepatic glands normal. Spleen normal in size; pulp rather soft, reddish-brown in colour, but apparently free from tubercles and degeneration. Some of the renal lobules appear normal, but the majority have larger or smaller areas, in some amounting to nearly half the lobule, in which the normal reddishbrown cortex is replaced by a dirty-white tissue, without any sign of degeneration. In some of the lobules this new tissue has invaded the boundary layer and medulla also, and in a few there are some welldefined white fibrous tubercles, about the size of a large pin's head, in the medulla and boundary layer. The mediastinal and bronchial lymphatic glands are enlarged to about double the normal size, and they are all crammed with tubercles, most of which are calcified. Both lungs contain a number of solid areas, the largest of which are adjacent to the thin lower edge, opposite the heart. On section these are found 
to contain caseous and partly calcified masses, varying in size from a hazel nut downwards. Elsewhere, throughout the spongy lung tissue, there are scattered firm, shot-like tubercles, but the distribution of these is not uniform. All the lymphatic glands of the head, neck, throat, and limbs, except those above-mentioned, are normal. The pia mater at the base of the brain, especially on the sides of the pons and medulla, shows some pin-head tubercles (tubercle bacilli readily found in these).

On the 24th December two-guinea-pigs were inoculated intraperitoneally with a trace of caseo-pus from the heifer's lung suspended in sterile water. One of these animals was found dead on the $23 \mathrm{rd}$ January, apparently in consequence of an accident, as the peritoneal cavity contained a large quantity of liquid blood. Numerous tubercles had developed in the omentum, and a few were also visible in the spleen. The other guinea-pig was killed on the 2 ist February, and the post-mortem examination showed the omentum converted into a solid mass with tubercles. The liver and spleen were greatly enlarged and filled with tubercies. A few tubercles were also present in the lungs.

\section{Case III.}

The subject in this case was a shorthorn cow, three years old when it was first brought under experiment on the 19th September I 898 . On that date it was tested with tuberculin and there was no reaction.

$\mathrm{Up}_{\mathrm{p}}$ to the date of the last report it had been five times ingculated intravenously with tubercle bacilli, as shown below :-

\begin{tabular}{|c|c|c|c|c|c|}
\hline Date. & \multicolumn{4}{|c|}{ Material used for Inoculation. } & Quantity. \\
\hline 1898 & \multirow{2}{*}{\multicolumn{4}{|c|}{ Emulsion of the teberculous liver of a fowl. }} & \\
\hline $\begin{array}{c}\text { Sept. } 29 \\
\text { I } 899\end{array}$ & & & & & $4 \mathrm{cc}$ \\
\hline $\begin{array}{c}\text { Nov. I I } \\
1900\end{array}$ & \multicolumn{4}{|c|}{ Same as used on that date for Case II. } & $4 \mathrm{cc}$ \\
\hline$\underset{\text { I }}{\text { Apo I }} 5$ & $"$ & " & , & Cases I. and II. & $3 \mathrm{cc} \cdot$ \\
\hline Mar. 23 & $"$ & $"$ & $"$ & Case II. & $\mathrm{I}_{5} \mathrm{cc}$ \\
\hline May i 8 & $"$ & $"$ & " & Cases I. and II. & $5 \mathrm{cc}$ \\
\hline
\end{tabular}

Subsequent to the date of the previous article the cow received three intravenous inoculations of virulent tubercle bacilli, as follows :-

Sixth Inoculation.-On the 3 Ist July $5 \mathrm{cc}$. of the liquid used on that date for the preceding two cases was injected into one of the jugular veins. An hour and a half later the temperature had risen to $105^{\circ} 5^{\circ}$, and it remained over $104^{\circ}$ for several days afterwards. Subsequently it fell to normal, and the cow appeared to be unaffected in any way by the inoculation. On the 13 th September it was tested with tuberculin, and the temperature rose from $1017^{\circ}$ to $103^{\circ}$ (9th hour).

Seventh Inoculation.-On the 5 th October the cow was inoculated intravenously with ro cc. of a rich emulsion of tubercle bacilli (the same as used on that date in Cases I. and II.), and a few hours after- 
wards the temperature had risen to $1045^{\circ}$ During the following week it varied from $101 \cdot 3^{\circ}$ to $103^{\circ} 8^{\circ}$, and then became normal.

The cow was tested with tuberculin on the dates and with the results shown below:-

Date. Time of Injection. 6th Hour. gth Hour. 12th Hour. 15th Hour.

$\begin{array}{llllll}22 / 10 / 01 & 1016 & 102.4 & \text { IOI'9 } & \text { IOI.6 } & \text { IO2 } \\ 29 / 11 / 01 & 101.4 & \text { IO2.8 } & \text { IO2.I } & \text { IOI. } 4 & \text { 10I.6 }\end{array}$

Eighth Inoculation.-On the 22 nd January last 4 cc. of a liquid made by rubbing up a quantity of caseo-purulent matter from the spleen of a horse with sterile water was injected into the cow's right jugular vein. On the following day the temperature was $103^{\circ}$, but subsequently it only once (8th February) exceeded $\mathrm{IO}^{\circ}$. The animal had fallen off to some extent in condition, although the respiratory movements were not sensibly disturbed, and the appetite was good.

On the morning of the loth February it was found that the cow was unable to rise, although she made strong efforts to do so with her fore quarters. Apparently in consequence of these efforts, the respirations were hurried, and the animal seemed excited. Up to this time she had been tied by the neck in a stall. With assistance she now managed to rise, walked a few yards to a loose-box, and immediately began to eat voraciously. Temperature $100^{\circ} 2^{\circ}$. The cow continued standing throughout the day, but on the following morning shewas found down in her box, with her neck extended on the ground, and her legs thrust out. Temperature $996^{\circ}$. As death appeared to be imminent, the animal was destroyed by chloroform, and the post-mortem examination, of which the following are the notes, was begun at once:-

Post-morten Examination.-Body in good condition; subcutaneous and intermuscular fat abundant; udder and its lymphatic glands normal.

Peritoneum normal; fat in omentum and mesentery and around kidneys very abundant. In the omentum, close to the fourth stomach, a mass of this fat as large as the two hands has quite an abnormal, firm consistence, feeling almost stony on manipulation, and contrasting with the soft, normal, semifluid consistence of the surrounding fat. On section this appears to be adipose tissue of which the oil has set during life (necrosis). (Microscopically this fat showed no gross abnormality.) There is a similar large mass in the fat around the right kidney, and several smaller ones in the fat around the large bowel.

One mesenteric lymphatic gland, somewhat smaller than a walnut, has a caseous, slightly gritty centre. Spleen and liver quite normal in size and appearance. In each kidney a number of the lobules have the cortex replaced by a greyish-pink new tissue, and the medulla of each of these lobules is destroyed by caseation. Pelvic organs normal.

Bronchial glands normal in size. The right contains several caseous tubercles, the largest the size of a pea. The left contains one such tubercle, the size of a barley grain. The mediastinal lymphatic gland is also normal in size, but it contains a considerable number (over a dozen) of caseous tubercles, some of them gritty, none larger than a pea. On the diaphragmatic and costal surface of the pleura there are some soft, villous, fleshy-looking growths, and there are 
some similar growths on the chest wall. In each lung there is a good deal of interlobular emphysema, and a few areas of collapse. Except for these areas of collapse, the lung tissue is everywhere spongy, but a few shot-like tubercles can be felt in it. These are caseous, and some of them are almost completely calcified. A few of them are pea-sized, but the majority are not larger than barley grains. In each lung they are most numerous towards the base.

With the exceptions already mentioned, the whole of the lymphatic glands of the body are normal.

In the upper fourth of the tongue, on its right lateral aspect, there is a shallow erosion of the mucous membrane, about half an inch in diameter, and very like a lesion of actinemycosis. When the tongue is cut across at this level it is found to have in its muscular substance a cavity nearly as large as a walnut, and filled with caseo-pus.

The membranes of the brain are normal, but the inferior face of the medulla oblongata, to the right of the mesial furrow, shows a diffuse, ill-defined swelling. On section, the normal tissye of the medulla is here seen to be replaced by a nodule of the size of a hazel nut, greyish in colour, but not visibly caseous.

Tubercle bacilli were readily found in scrapings from the lesions in the tongue and medulla oblongata.

In reviewing the results of these experiments the first question that naturally presses for an answer is, Did the animals in the course of the experiments acquire an increased power of resisting infection with tubercle bacilli? In spite of the fact that each of the animals ultimately succumbed to tuberculosis, it will scarcely be denied by anyone that in the course of the experiments they all displayed a remarkable degree of immunity, but it might be suggested that this immunity was in each case natural. The suggestion is within the bounds of possibility, but it does not appear to be at all probable. My own previous experiments ${ }^{1}$ indicate that the majority of cattle will promptly succumb to the first intravenous injection of tubercle bacilli, even when the quantity injected is very much smaller than was employed in the cases described in this article. At the same time it must be admitted that the degree of resistance which animals of the bovine species naturally offer to infection with tuberculosis varies considerably. The control calf of Case I (p. 63) appears to be an example of powers of resistance much above the average. ${ }^{2}$

In connection with this point, it is important to note that none of the bacilli employed in these experiments were derived from human lesions. The artificial cultures used were of two different strains, one obtained directly from a natural case of tuberculosis in a dog, and the other by passage through the rabbit from a natural case of the disease in a sheep. Control experiments showed that these cultures were intensely virulent for rodents when they were used for the intravenous inoculation of the cattle in the first half of I9OI, and they still possessed what may be called average virulence at the dates of the later inoculations.

It therefore appears to be justifiable to conclude that, whatever

1 See this "Journal," Vol. XI, p. 244, and Vol. XIII, p. 368, et seq.

2 It ought perhaps to be admitted as possible that at the inoculation of this animal a considerable proportion of the liquid escaped between the skin and the vein. 
may have been the degree of natural immunity possessed by these three experimental cattle, it was much increased by the successive intravenous inoculations to which they were subjected. The immunity was not absolute, but it may be doubted whether a degree of resistance that would merit that term is obtainable by any method in cattle.

A very singular feature in connection with these experiments is the distribution of the lesions found at the post-mortem examination of each of the three animals. Space will not permit me to consider these peculiarities at length on this occasion, but attention may be called to the absence of lesions from the spleen and liver in each case, the constancy of lesions in the kidneys, the occurrence of tuberculosis of the pia mater in two of the animals, the implication of the tongue and medulla oblongata in the third, the presence of tubercles in various groups of peripheral lymphatic glands in one, and the escape of the same parts in the other two animals.

Lastly, the way in which the animals behaved to the various tests with tuberculin merits attention. On a good many of the occasions there was no reaction at all, but in a considerable number there was a slight rise of temperature, and in some the rise was sufficient to constitute a distinct reaction. As a rule, when there was only a slight rise, and sometimes even when there was a distinct reaction, the maximum temperature was reached at the sixth or ninth hour. The rise was thus on many occasions not of the type which is generally considered characteristic of a reaction to tuberculin, but, nevertheless, it is very probable that it was a true reaction, in the sense that it was dependent upon the existence (sometimes temporary) of active tuberculous lesions within the body. Similar early rises of temperature are every now and again met with in ordinary practice when herds are tested with tuberculin, and it is very far from certain that in such cases it is safe to consider the animal not tuberculous because the temperature has' not been what some authors call typical.

\section{EDITORIAL ARTICLE.}

\section{$\longrightarrow 0-$ \\ THE SPONTANEOUS ORIGIN OF SWINE-FEVER.}

IN an article which appeared in the preceding number of this fournal it was pointed out that in the opinion of $\mathrm{Mr}$ Hanbury, President of the Board of Agriculture, the veterinary profession had yet to learn the A B C of swine-fever. Stimulated, apparently, by further reflection on the ignorance of the class to whom the public naturally turn for information regarding this and other diseases of animals, the President has himself made an incursion into the field of veterinary pathology, and the first fruits of his researches were communicated to the members of the Taunton Farmers' Club on the first March 